September 2014, Volume 2

Proceeding of $3^{\text {rd }}$ International Conference on Computation for Science and Technology, ISSN xxxx-yyyy

\title{
Structural Study of Chalcogenide Material Ge-Te-Ga using ab Initio Molecular Dynamics
}

\author{
Lilin Lalita $^{1 *}$, Hermawan K. Dipojono ${ }^{1}$, Muhamad A.Martoprawiro ${ }^{2}$ \\ ${ }^{1}$ Department of Engineering Physics, Faculty of Industrial Engineering, Bandung Institute of Technology, Jl. Ganesha 10, Bandung 40132, \\ Indonesia. \\ ${ }^{2}$ Department of Computational Science, FMIPA, Bandung Institute of Technology, Jalan Ganesha 10, Bandung 40132, Indonesia.
}

Received: 20 September 2014 / Accepted: 30 November 2014

\begin{abstract}
:
Due to its capability of quick amorphous-crystalline phase transition, chalcogenide material Ge-Te-Ga is a potential candidate of a phase change material. For the amorphous phase, the dominant bonding is between Ga and Te. The first peak of Ga-Te bonds is at about $2.7 \AA$. In the liquid phase, on the other hand, the dominant bonding is between Ge and Te, i.e. at about $2.8 \AA$. Atoms tend to bind the system in heteropolar. The results of the calculation of the radial distribution function showed that the amorphous phase had a degree of regularity which was better than the amorphous phase. This was consistent with the calculation results of the distribution of bond angles. In addition, the calculation of the distribution of bond angles also indicated that the system was mostly tetrahedral and structurally defective octahedral. Statistical analysis of rings revealed that all samples of amorphous phase had dominant fourfold ring. Formation of rings was a basic process causing the crystallization took place very fast.
\end{abstract}

Key words: ab initio, chalcogenide, crystallization, molecular dynamics, phase change material

\section{Introduction}

Today, lifestyle paradigm shifts to the mobile lifestyle. With mobile lifestyle, the needs of data storage media will continue to rise. As result, data storage media which are faster, smaller,more efficient and capable of storing more data than the previous knowndata storage mediaare required both for personal purposes as well as for largescale. One solution offered as an alternative for data storage technology is phase change memory. The mechanism of phase change memory is based on optical and electronics properties difference between amorphous and crystalline phases [1]. Chalcogenide material is a common material for phase change memory due to its unique properties that can change from amorphous to crystalline phases and vice versa [2]; due to this unique properties, chalcogenide material is known as phase change material [3].

Although the application of chalcogenide materials as phase change materials have been found more than thirty years ago, but the mechanism of amorphous-crystalline phase transition and why thistransition occurs rapidly is still unknown until today $[4,5,6,7]$. To answer how the mechanism of phase changes in the phase change material a study of atomics structure is required. At the atomic scale, the phase change mechanism will be numerically simulated using $a b$ initio molecular dynamics simulation approach.

\footnotetext{
*Corresponding author: Lilin Lalita,
}

E-mail: lilinlalita@gmail.com

\section{Experimental}

In this study the structure of chalcogenide material GeTe4 with gallium as a dopant $(0,10$, and 20 in $\%)$ was generated using $a b$ initio molecular dynamics method. The software used for the simulation is SIESTA [10] using a linear combination of numerical atomic orbitals as the basis set and norm-conserving pseudopotentials. The total energy is approximated in the non-selfconsistent Harris functional.

The number of atoms used is $80-100$ atoms. The atom is randomly inside cubic lattice with boundary and lattice constant depend on system mass density from experimental results [11]. Modelling amorphous and liquid phases will be conducted by using melt quenching technique. There are two main processes carried out in the melt quenching technique namely processes NVT molecular dynamics (at a temperature of $1400 \mathrm{~K}$ and 300 $\mathrm{K}$ ) and annealing for cooling (from a temperature of $1400 \mathrm{~K}$ to $300 \mathrm{~K}$ ). Further crystallization modeling material made of Ge-Te-Ga consisting of five stages: a three-stage process of NVT $a b$ initio molecular dynamics (at a temperature of $600 \mathrm{~K}, 550 \mathrm{~K}$, and $500 \mathrm{~K}$ ) and two-stages annealing with slow cooling rates (of temperature $600 \mathrm{~K}$ to $550 \mathrm{~K}$ temperature obvious even $550 \mathrm{~K}$ to $500 \mathrm{~K}$ ). 
Coordination number, radial distribution functions, structure factors, the distribution of bond angles, and ring statistics are structural parameters that will be examined in this study. The material crystallization of Ge-Te-Ga done visually by looking at the formation of the crystalline structure of the amorphous network.

\section{Results and Discussion}

The structure of Ge-Te-Ga has been obtained using $a b$ initio molecular dynamics simulation. Figure 1.(a,b,c) shows 100 atoms configurarion of amorphous Ge-Te-Ga which each atoms displayed by yellow for germanium, purple for tellurium, and pink for gallium. Figure $1(\mathrm{~d}, \mathrm{e}, \mathrm{f})$. shows distribution of coordination number of Ge-Te-Ga system (turquoise: 1, burlywod: 2, Forest green: 3, grey: 4, red: 5, brown: 6, purple: 7). It is known that amorphous Ge-Te-Ga is dominated by atoms with coordination number 3 and 4 .
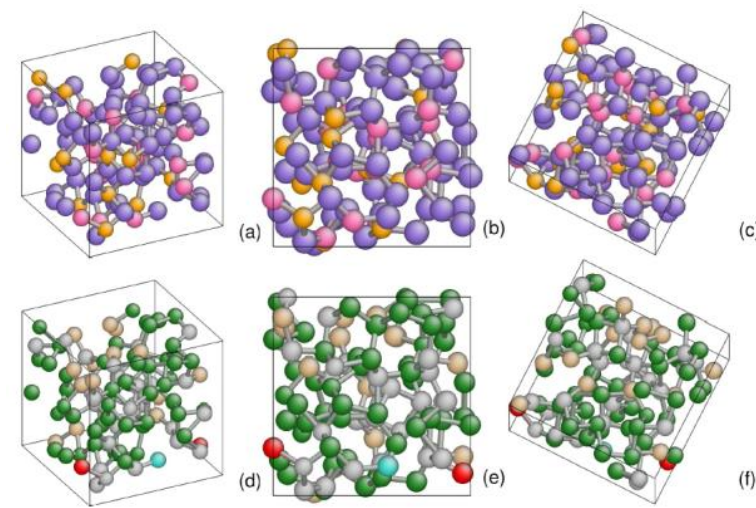

Figure 1. Atom configuration and coordination number of GeTe-Ga system.

Radial distribution function (RDF) is one of important parameter to study structural characteristics, RDF is key to investigate especially short and medium range order of material. The method can be applied to amorphous, liquid, and crystalline.

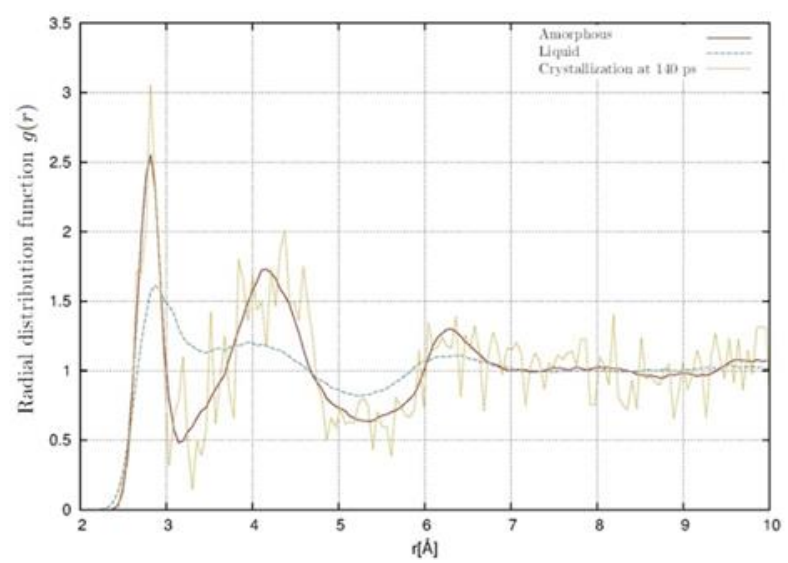

Figure 2. Radial distribution function of Ge-Te-Ga system.

$\mathrm{Ge}-\mathrm{Te}-\mathrm{Ga}$ in liquid phase has wider radial distribution function and lower peak intensity compared to Ge-Te-Ga system in amorphous phase, as shown in figure 2. For the amorphous phase, the dominant bonding is Ga-Te. The first peak of Ga-Te bonds are at about $2.7 \AA$. In the liquid phase, on the other hand, the dominant bonding is Ge-Te, i.e. at about $2.8 \AA$. Atoms tend to bind the system in heteropolar. The results of the calculation of the radial distribution function shows that the amorphous phase has a degree of regularity which is better than the amorphous phase.

Bond angle distribution between first neighbor atoms can give information about structural nature of system. As member of group IV Chalcogenide, we expect that $\mathrm{GeTe}_{4}$ system has tetrahedral nature as $\mathrm{GeSe}_{4}$.

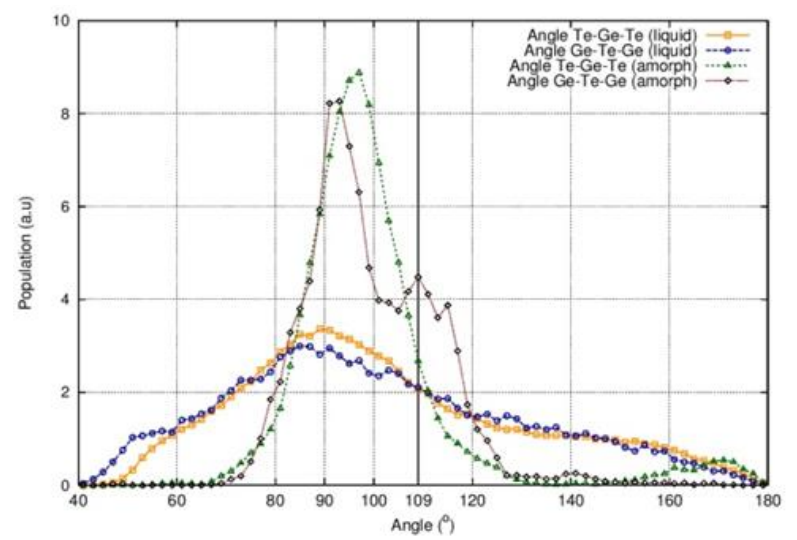

Figure 3. Bond angle distribution of Ge-Te-Ga system.

The average of coordination number of Ge in amorphous phase is about 4. However it doesn't prove that amorphous phase only has tetrahedral coordination. Figure 3 gives information that Ge-Te-Ga system has maximum bond angle about $88^{\circ}-100^{\circ}$. From the given information, we know that Ge-Te-Ga system should be made of fourfold rings which is mostly tetrahedral and octahedral structurally defective.

As expected, the calculation of ring statistic has suitability with bond angle distribution analysis. The structure of Ge-Te-Ga system shows that amorphous GeTe-Ga has significant number of fourfold rings which mostly in $\mathrm{ABAB}$ form $(\mathrm{A}=\mathrm{Ge}, \mathrm{Ga} ; \mathrm{B}=\mathrm{Te})$. The number of fourfold rings increases for amorphous phase. From the calculation of ring statistics and bond angle distribution, it is known that Ge-Te-Ga system has similar characteristics with Ge-Sb-Te system [9]. It can be assumed that fourfold rings has responsibility in rapid amorphous-crystalline phase transition.

\section{Conclusions}

Calculation result of bond angle distributions suggests that the structure of Ge-Te-Ga should be made of fourfold rings which is partially tetrahedral and defective octahedral like. The amorphous Ge-Te-Ga systems have a significant number of fourfold rings which responsible to the rapid crystal growth in crystal-amorphous transition. 


\section{References}

[1] M. Wuttig and M. Anbarasu, Understanding the structure and properties of phase change materials for data storage applications, Journal of the Indian Institute of Science, 91(21), 2011, 259.

[2] S. Mittal, Energy saving techniques for phase change memory (pcm), arXiv:1309.3785, 2013.

[3] S.R. Ovshinsky, Reversible electrical switching phenomena in disordered structures, Phys. Rev. Lett., 21, 1968, 14501453.

[4] V. Sousa, Chalcogenide materials and their application to non-volatile memories, Microelectronic Engineering, 88, 2011, 807-813.

[5] I. Tortorelli, A. Redaelli, A. Pirovano, M. Allegra, M. Magistretti, C. Bresolin, D. Erbetta, A. Modelli, E. Varesi, F. Pellizzer, A. L. Lacaita, R. Bez, M. Boniardi, and D. Ielmini, Impact of Ge-Sb-Te compound engineering on the set operation performance in phase-change memories, SolidState Electronics, 58, 2011, 11-16.
[6] A.I. Popov, S.A. Kozyukhin, and E.N. Voronkov, Influence of Chalcogenide glasses electro physical parameters on threshold voltage for phase-change memory, Thin Solid Films, 518, 2010, 5656-5658.

[7] M. Skapas, C. Wiemer, O. Salicio, M. Longo, A. Pirovano, J. Siegel, W. Gawelda, S. Rushworth, C. Giesen, A. Abrutis, and V. Plausinaitiene, Chemical vapor deposition of chalcogenide materials for phase-change memories, Microelectronic Engineering, 85, 2008, 2338-2341.

[8] S.R. Elliot, Physics of Amorphous Materials, Longman, New York, 1990.

[9] S.R. Elliot and J. Hegedus, Microscopic origin of the fast crystallization ability of $\mathrm{Ge}-\mathrm{Sb}$-Te phase change memory materials, Nature Mat., 7, 2008, 399.

[10] D. Lencer, M. Salinga, B. Grabowski, T. Hickel, 11. J. Neugenauer, and M. Wuttig, Nature Mat., 7, 2008, 972.

[11] V. Ilcheva, T. Petkova, P. Petkov, and P. Ilchev, Physiochemical properties of Ge-Te-Ga glasses, J. Optoel. Adv. Mat., 9, 2007, 3093. 